VoL. 57 (1998) [253-259]

\title{
SHARP GRADIENT ESTIMATES FOR EIGENFUNCTIONS ON RIEMANNIAN MANIFOLDS
}

\section{ALBERT BORBÉLY}

Sharp gradient estimates are derived for positive eigenfunctions on complete Riemannian manifolds with Ricci curvature bounded below.

\section{INTRODUCTION}

The aim of this note is to derive, in a simple and rather elementary way, sharp gradient estimates for positive eigenfunctions on complete Riemannian manifolds with Ricci curvature bounded from below by a constant. One can view these inequalities as the infinitesimal form of the Harnack inequality for positive eigenfunctions on complete Riemannian manifolds.

Estimates of this type were obtained in [2]. In case of harmonic functions the estimate is sharp [2, Theorem $\left.3^{\prime \prime}\right]$, but for eigenfunctions in general [2, Theorem $\left.3^{\prime}\right]$ the constant involved in the estimate was not explicitely computed.

For an eigenfunction $f$ with a negative eigenvalue, a sharp lower bound on the $\sup |\nabla f| / f$ is obtained as well.

The main result is the following.

ThEOREM. Let $M=M^{n}$ be an $n$-dimensional complete Riemannian manifold with Ricci curvature bounded below by $-(n-1) K^{2},(K \geqslant 0)$ and let $f$ be a positive eigenfunction, that is, $\Delta f=\lambda f$ for some $\lambda$. Then $\lambda \geqslant-(1 / 4)(n-1)^{2} K^{2}$ and

$$
\frac{(n-1) K-\sqrt{((n-1) K)^{2}+4 \lambda}}{2} \leqslant \sup \frac{|\nabla f|}{f} \leqslant \frac{(n-1) K+\sqrt{((n-1) K)^{2}+4 \lambda}}{2} .
$$

$\begin{aligned} \text { If, at some point } q,|\nabla f| / f & =\sup |\nabla f| / f \text { and }|\nabla f| / f=(1 / 2)((n-1) K \\ \left.+\sqrt{((n-1) K)^{2}+4 \lambda}\right) & \text { or }|\nabla f| / f=(1 / 2)\left((n-1) K-\sqrt{((n-1) K)^{2}+4 \lambda}\right) \text { with }\end{aligned}$ $\lambda<0$, then, at this point $\operatorname{Ric}((\nabla f) /|\nabla f|,(\nabla f) /|\nabla f|)=-(n-1) K^{2}$ and $\nabla f$ is an eigenvector and $\nabla f^{\perp}$ is an eigenspace of Hess $f$.

To see that the estimates above are sharp, one takes the constant curvature model $M_{K}$, a simply connected space of constant sectional curvature $-K^{2}$. Set

Received 15th July, 1997

Copyright Clearance Centre, Inc. Serial-fee code: 0004-9729/98 \$A2.00+0.00. 
$f=\exp (-\alpha b)$, where $b$ denotes a Busemann function on $M_{K}$. Then, a simple computation shows that $f$ is an eigenfunction, with eigenvalue $\lambda=\alpha^{2}-\alpha(n-1) K$ and equality is achieved in the above inequalities.

The situation is quite different if the Ricci curvature is non-negative. From the proof of the Theorem one obtains easily the following.

Corollary. Let $M=M^{n}$ be an n-dimensional complete Riemannian manifold with Ricci curvature bounded below by $C>0$. Then there is no positive function $f$ defined on $M$ with $\Delta f=\lambda f$ for any $\lambda \neq 0$. If $\lambda=0$, then the only solution of $\Delta f=\lambda f$ is the constant function.

The case of harmonic functions $(\lambda=0)$ was already covered in [2] but we added it for the sake of completeness.

\section{Preliminaries}

For the convenience of the reader, in this section, we collect some basic formulas which will be used later on.

Let $M=M^{n}$ be an $n$-dimensional complete Riemannian manifold. Then, for any $f \in C^{\infty}(M)$ the Bochner-Lichnerowicz formula [1, Propisition 4.15] states that

$$
\frac{1}{2} \Delta\left(|\nabla f|^{2}\right)=|\operatorname{Hess} f|^{2}+\langle\nabla f, \nabla(\Delta f)\rangle+\operatorname{Ric}(\nabla f, \nabla f)
$$

For a linear map $A: T_{p} M \rightarrow T_{p} M$ we define $|A|^{2}$ by

$$
|A|^{2}=\operatorname{tr} A^{T} A=\sum_{i=1}^{n}\left|A E_{i}\right|^{2}=\sum_{i, j=1}^{n}\left\langle A E_{i}, E_{j}\right\rangle^{2}
$$

for any orthonormal system $E_{1}, \ldots, E_{n}$.

The following elementary estimate will play an important role.

Proposition 1.1. Let $U \in T_{p} M$ be any unit vector and set $\operatorname{tr} A=\lambda$. Then we have

$$
|A|^{2} \geqslant\langle A U, U\rangle^{2}+\frac{1}{n-1}(\langle A U, U\rangle-\lambda)^{2}
$$

Equality occurs if and only if $U$ is an eigenvector and $U^{\perp}$ is an eigenspace.

Proof: Let $U=E_{1}, E_{2}, \ldots, E_{n}$ be an orthonormal system. Then, we have

$$
\operatorname{tr}(A)=\sum_{i=1}^{n}\left\langle A E_{i}, E_{i}\right\rangle=\lambda
$$


By the Cauchy-Schwarz inequality we see that

$$
\left(\left\langle A E_{1}, E_{1}\right\rangle-\lambda\right)^{2} \leqslant(n-1) \sum_{i=2}^{n}\left\langle A E_{i}, E_{i}\right\rangle^{2} .
$$

Adding $(n-1)\left\langle A E_{1}, E_{1}\right\rangle^{2}$ to both sides we get that

$$
(n-1)\left\langle A E_{1}, E_{1}\right\rangle^{2}+\left(\left\langle A E_{1}, E_{1}\right\rangle-\lambda\right)^{2} \leqslant(n-1) \sum_{i=1}^{n}\left\langle A E_{i}, E_{i}\right\rangle^{2} \leqslant(n-1) \mid A^{2} .
$$

From this the proposition follows easily.

The case of equality is simple as well. From the last inequality we conclude that $E_{1}, \ldots, E_{n}$ must all be eigenvectors and the equality case of Cauchy- Schwarz implies that $E_{2}, \ldots, E_{n}$ must have the same eigenvalues. This concludes the proof.

We also need a formula for $\mid$ Hess $\left.\log f\right|^{2}$. From (1.1) we have

$$
\begin{aligned}
\mid \text { Hess }\left.\log f\right|^{2} & =\sum_{i, j=1}^{n}\left\langle\nabla_{E_{i}} \frac{\nabla f}{f}, E_{j}\right\rangle^{2}=\sum_{i, j=1}^{n}\left(\frac{1}{f}\left\langle\nabla_{E_{i}} \nabla f, E_{j}\right\rangle-\frac{1}{f^{2}}\left\langle E_{i} f \nabla f, E_{j}\right\rangle\right)^{2} \\
& =\sum_{i, j=1}^{n} \frac{1}{f^{2}}\left\langle\nabla_{E_{i}} \nabla f, E_{j}\right\rangle^{2}+\frac{1}{f^{4}}\left\langle E_{i} f \nabla f, E_{j}\right\rangle^{2}-\frac{2}{f^{3}}\left\langle\nabla_{E_{i} f E_{i}} \nabla f, E_{j} f E_{j}\right\rangle .
\end{aligned}
$$

Hence we have

$$
\mid \text { Hess }\left.\log f\right|^{2}=\frac{\mid \text { Hess }\left.f\right|^{2}}{f^{2}}+\left|\frac{\nabla f}{f}\right|^{4}-2 \frac{\operatorname{Hess} f}{f}\left(\frac{\nabla f}{f}, \frac{\nabla f}{f}\right) .
$$

We now establish the crucial inequality about eigenfunctions.

Proposition 1.2. Let $M^{n}$ be an $n$-dimensional complete Riemannian manifold and let $f$ be a positive eigenfunction with $\Delta f=\lambda f$. Then

$$
\begin{aligned}
\frac{n-1}{2} \Delta\left|\frac{\nabla f}{f}\right|^{2} \geqslant( & \left.\left\langle\frac{\nabla f}{|\nabla f|}, \frac{\nabla\left(\frac{1}{2}|\nabla f|^{2}\right)}{|\nabla f| f}\right\rangle-\lambda\right)^{2}+(n-1) \operatorname{Ric}\left(\frac{\nabla f}{f}, \frac{\nabla f}{f}\right) \\
& +2(n-1)\left|\frac{\nabla f}{f}\right|^{2}\left(\left|\frac{\nabla f}{f}\right|^{2}-\left\langle\frac{\nabla f}{|\nabla f|}, \frac{\nabla\left(\frac{1}{2}|\nabla f|^{2}\right)}{|\nabla f| f}\right\rangle\right) .
\end{aligned}
$$

If at some point $p \in M^{n}$ equality occurs then at that point $\nabla f$ is an eigenvector and $\nabla f^{\perp}$ is an eigenspace of Hess $f$.

ProOF: First we apply the Bochner-Lichnerowicz formula to $\log f$.

$\frac{1}{2} \Delta\left(|\nabla \log f|^{2}\right)=\mid$ Hess $\left.\log f\right|^{2}+\langle\nabla \log f, \nabla(\Delta \log f)\rangle+\operatorname{Ric}(\nabla \log f, \nabla \log f)$. 
Taking into account (1.2) and the following simple identities

$$
\Delta f=\lambda f, \quad \nabla \log f=\frac{\nabla f}{f}, \quad \Delta \log f=\frac{\Delta f}{f}-\left|\frac{\nabla f}{f}\right|^{2} .
$$

we get

$$
\begin{aligned}
\frac{1}{2} \Delta\left|\frac{\nabla f}{f}\right|^{2}= & \frac{\mid \text { Hess }\left.f\right|^{2}}{f^{2}}+\left|\frac{\nabla f}{f}\right|^{4}-2 \frac{\text { Hess } f}{f}\left(\frac{\nabla f}{f}, \frac{\nabla f}{f}\right) \\
& -2\left\langle\frac{\nabla f}{f}, \nabla\left(\frac{\frac{1}{2}|\nabla f|^{2}}{f^{2}}\right)\right\rangle+\operatorname{Ric}\left(\frac{\nabla f}{f}, \frac{\nabla f}{f}\right) \\
= & \frac{\mid \text { Hess }\left.f\right|^{2}}{f^{2}}+3\left|\frac{\nabla f}{f}\right|^{4}-2 \frac{\text { Hess } f}{f}\left(\frac{\nabla f}{f}, \frac{\nabla f}{f}\right) \\
& -2\left\langle\frac{\nabla f}{f}, \frac{\nabla\left(\frac{1}{2}|\nabla f|^{2}\right)}{f^{2}}\right\rangle+\operatorname{Ric}\left(\frac{\nabla f}{f}, \frac{\nabla f}{f}\right) .
\end{aligned}
$$

A simple computation shows that

$$
\frac{\text { Hess } f}{f}\left(\frac{\nabla f}{|\nabla f|}, \frac{\nabla f}{|\nabla f|}\right)=\left\langle\frac{\nabla f}{|\nabla f|}, \frac{\nabla\left(\frac{1}{2}|\nabla f|^{2}\right)}{|\nabla f| f}\right\rangle
$$

and

$$
\frac{\operatorname{Hess} f}{f}\left(\frac{\nabla f}{f}, \frac{\nabla f}{f}\right)=\left|\frac{\nabla f}{f}\right|^{2}\left\langle\frac{\nabla f}{|\nabla f|}, \frac{\nabla\left(\frac{1}{2}|\nabla f|^{2}\right)}{|\nabla f| f}\right\rangle
$$

Applying now Proposition 1.1 to (Hess $f$ ) $/ f$ we have

$$
\begin{aligned}
\frac{1}{2} \Delta\left|\frac{\nabla f}{f}\right|^{2} \geqslant & \left(\left\langle\frac{\nabla f}{|\nabla f|}, \frac{\nabla\left(\frac{1}{2}|\nabla f|^{2}\right)}{|\nabla f| f}\right\rangle^{2}+\left|\frac{\nabla f}{f}\right|^{4}-2\left\langle\frac{(\nabla f)|\nabla f|}{f^{2}}, \frac{\nabla\left(\frac{1}{2}|\nabla f|^{2}\right)}{|\nabla f| f}\right\rangle\right) \\
& +\frac{1}{n-1}\left(\left\langle\frac{\nabla f}{|\nabla f|}, \frac{\nabla\left(\frac{1}{2}|\nabla f|^{2}\right)}{|\nabla f| f}\right\rangle-\lambda\right)^{2}+\operatorname{Ric}\left(\frac{\nabla f}{f}, \frac{\nabla f}{f}\right) \\
& +2\left|\frac{\nabla f}{f}\right|^{2}\left(\left|\frac{\nabla f}{f}\right|^{2}-\left\langle\frac{\nabla f}{|\nabla f|}, \frac{\nabla\left(\frac{1}{2}|\nabla f|^{2}\right)}{|\nabla f| f}\right\rangle\right) .
\end{aligned}
$$

It is easy to see that on the right hand side the first term in parenthesis is always non-negative, therefore we have

$$
\begin{aligned}
\frac{1}{2} \Delta\left|\frac{\nabla f}{f}\right|^{2} \geqslant & \frac{1}{n-1}\left(\left\langle\frac{\nabla f}{|\nabla f|}, \frac{\nabla\left(\frac{1}{2}|\nabla f|^{2}\right)}{|\nabla f| f}\right\rangle-\lambda\right)^{2}+\operatorname{Ric}\left(\frac{\nabla f}{f}, \frac{\nabla f}{f}\right) \\
& +2\left|\frac{\nabla f}{f}\right|^{2}\left(\left|\frac{\nabla f}{f}\right|^{2}-\left\langle\frac{\nabla f}{|\nabla f|}, \frac{\nabla\left(\frac{1}{2}|\nabla f|^{2}\right)}{|\nabla f| f}\right\rangle\right) .
\end{aligned}
$$

This implies the proposition. The case of equality follows easily from the equality case of Proposition 1.1. 


\section{Proof of the Theorem.}

Let $M=M^{n}$ be an $n$-dimensional complete Riemannian manifold with Ricci curvature bounded below by $-(n-1) K^{2}$ and let $f$ be a positive eigenfunction, that is, $\Delta f=\lambda f$ for some $\lambda$. By [2, Theorem $\left.3^{\prime}\right]$ we know that $|\nabla f| / f$ is bounded. Set

$$
\alpha=\sup \frac{|\nabla f|}{f} \text {. }
$$

For any $0<\varepsilon<1$ there is a point $q \in M$ such that

$$
\frac{|\nabla f|}{f}(q)>\alpha(1-\varepsilon) \text {. }
$$

For some $\delta>0$ (which will be choosen later) let $h: \mathbb{R}^{+} \rightarrow \mathbb{R}^{+}$be a smooth, decreasing function such that $h(t)=1$ for $0 \leqslant t<1, \lim _{t \rightarrow \infty} h(t)=1-\varepsilon$ and $\left|h^{\prime}\right|,\left|h^{\prime \prime}\right|<$ $\delta$. Clearly such a function must exist.

Let $g: M \rightarrow \mathbb{R}^{+}$be defined by $g(p)=h\left(d_{q}(p)\right)$, where $d_{q}$ is the distance function from $q$. From [2, Lemma 1] we know that $\Delta d_{g}<C$ for $d_{g}>1$, where $C$ is some constant depending only on the dimension and the lower bound on the Ricci curvature. Taking into account that $\Delta g=h^{\prime} \Delta d_{q}+h^{\prime \prime}\left|\nabla d_{q}\right|^{2}$, for a small enough $\delta$ we get that

$$
\frac{|\nabla g|}{g}<\varepsilon, \quad \frac{\Delta g}{g}>-\varepsilon
$$

Consider now the function $g|\nabla f / f|^{2}$. This must assume its maximum at some point $q^{\prime}$. Hence, at the point $q^{\prime}$, we have

$$
\nabla\left(g\left|\frac{\nabla f}{f}\right|^{2}\right)=0, \quad \Delta\left(g\left|\frac{\nabla f}{f}\right|^{2}\right) \leqslant 0, \quad \text { and } \quad\left|\frac{\nabla f}{f}\right|^{2} \geqslant \alpha^{2}(1-\varepsilon)^{2} .
$$

From the first equality we get

$$
\nabla\left(\left|\frac{\nabla f}{f}\right|^{2}\right)=-\frac{\nabla g}{g}\left|\frac{\nabla f}{f}\right|^{2} .
$$

Applying this, the first inequality in (2.2) yields

$$
\Delta\left(\left|\frac{\nabla f}{f}\right|^{2}\right) \leqslant\left(2\left|\frac{\nabla g}{g}\right|^{2}-\frac{\Delta g}{g}\right)\left|\frac{\nabla f}{f}\right|^{2} .
$$

Combining (2.1) and Proposition 1.2 we get that

$$
\begin{aligned}
\frac{n-1}{2}\left(2 \varepsilon^{2}+\varepsilon\right)\left|\frac{\nabla f}{f}\right|^{2} \geqslant & \left(\left\langle\frac{\nabla f}{|\nabla f|}, \frac{\nabla\left(\frac{1}{2}|\nabla f|^{2}\right)}{f|\nabla f|}\right\rangle-\lambda\right)^{2}+(n-1) \operatorname{Ric}\left(\frac{\nabla f}{f}, \frac{\nabla f}{f}\right) \\
& +2(n-1)\left|\frac{\nabla f}{f}\right|^{2}\left(\left|\frac{\nabla f}{f}\right|^{2}-\left\langle\frac{\nabla f}{|\nabla f|}, \frac{\nabla\left(\frac{1}{2}|\nabla f|^{2}\right)}{|\nabla f| f}\right\rangle\right),
\end{aligned}
$$


at the point $q^{\prime}$. From (2.3) we also have

$$
\frac{\nabla\left(\frac{1}{2}|\nabla f|^{2}\right)}{f|\nabla f|}=\frac{\nabla f}{f} \frac{|\nabla f|}{f}-\frac{\nabla g}{g}\left|\frac{\nabla f}{f}\right|^{2} .
$$

Substituting this into the above inequality and using the fact that the Ricci curvature is bounded below by $-(n-1) K^{2}$, we have (at the point $q^{\prime}$ )

$$
\left(\left(1-\varepsilon_{1}\right)\left|\frac{\nabla f}{f}\right|^{2}-\lambda\right)^{2} \leqslant(n-1)^{2} K^{2}\left|\frac{\nabla f}{f}\right|^{2}+\varepsilon_{2}^{2}\left|\frac{\nabla f}{f}\right|^{2},
$$

where $\varepsilon_{1}=\langle\nabla f, \nabla g\rangle /(|\nabla f| g)$ and $\varepsilon_{2}=\sqrt{((n-1) / 2)\left(2 \varepsilon^{2}+\varepsilon\right)+2(n-1) \varepsilon \alpha^{2}}$. It is clear from (2.1) that both $\varepsilon_{1}$ and $\varepsilon_{2}$ approach 0 when $\varepsilon$ does.

Taking square roots, we have

$$
\left(1-\varepsilon_{1}\right)\left|\frac{\nabla f}{f}\right|^{2}-\lambda \leqslant\left((n-1) K+\varepsilon_{2}\right) \frac{|\nabla f|}{f} .
$$

This implies that at the point $q^{\prime}$

$$
\frac{|\nabla f|}{f} \geqslant \frac{(n-1) K+\varepsilon_{2}-\sqrt{\left((n-1) K+\varepsilon_{2}\right)^{2}+4 \lambda}}{2\left(1-\varepsilon_{1}\right)}
$$

and, by the last inequality of $(2.2)$,

$$
\alpha(1-\varepsilon) \leqslant \frac{|\nabla f|}{f} \leqslant \frac{(n-1) K+\varepsilon_{2}+\sqrt{\left((n-1) K+\varepsilon_{2}\right)^{2}+4 \lambda}}{2\left(1-\varepsilon_{1}\right)} .
$$

Now, the inequalities in the theorem follow by letting $\varepsilon$ go to 0 . Let us remark that the same argument also shows that $\lambda \geqslant-(1 / 4)(n-1)^{2} K^{2}$.

If, at some point $q \in M,|\nabla f| / f=\sup |\nabla f| / f=\alpha$, then at this point we have

$$
\nabla\left(\left|\frac{\nabla f}{f}\right|^{2}\right)=0 \text { and } \Delta\left(\left|\frac{\nabla f}{f}\right|^{2}\right) \leqslant 0 .
$$

From the first equality we get

$$
\frac{\nabla\left(\frac{1}{2}|\nabla f|^{2}\right)}{f|\nabla f|}=\frac{\nabla f}{f} \frac{|\nabla f|}{f}
$$

Combining these with Proposition 1.2, we have (at the point $q$ )

$$
\left(\alpha^{2}-\lambda\right)^{2}+\alpha^{2}(n-1) \operatorname{Ric}\left(\frac{\nabla f}{|\nabla f|}, \frac{\nabla f}{|\nabla f|}\right) \leqslant 0 .
$$


Now, if $\alpha=(1 / 2)\left((n-1) K+\sqrt{((n-1) K)^{2}+4 \lambda}\right)$ or $\alpha=(1 / 2)((n-1) K-$ $\left.\sqrt{((n-1) K)^{2}+4 \lambda}\right)$ with $\lambda<0$, then a simple computation shows that the inequality above can hold only if, at the point $q$, Ric $((\nabla f) /|\nabla f|,(\nabla f) /|\nabla f|)=-(n-1) K^{2}$. In this case, the inequality becomes an equality and the Theorem follows from the equality case of Proposition 1.2.

Proof of the Corollary: Up to (2.4) the argument is the same as in the proof of the Theorem. But if the Ricci curvature is bounded below by $C>0$, then the inequality (2.4) will be the following. At the point $q^{\prime}$ we have

$$
\left(\left(1-\varepsilon_{1}\right)\left|\frac{\nabla f}{f}\right|^{2}-\lambda\right)^{2} \leqslant-C\left|\frac{\nabla f}{f}\right|^{2}+\varepsilon_{2}^{2}\left|\frac{\nabla f}{f}\right|^{2}
$$

In case $\lambda \neq 0$, for a small enough $\varepsilon$ it is clearly impossible. If $\lambda=0$, then, by letting $\varepsilon$ go to 0 , we conclude that $\sup |\nabla f| / f=0$. This completes the proof of the Corollary. $\square$

\section{REFERENCES}

[1] S. Gallot, D. Hulin and J. Lafontaine, Riemannian geometry (Springer-Verlag, Berlin, Heidelberg, New York, 1993).

[2] S.T. Yau, 'Harmonic functions on complete Riemannian manifolds', Comm. Pure Appl. Math. 28 (1975), 201-228.

Department of Mathematics and Computer Science

Kuwait University

PO Box 5969

Safat 13060

Kuwait 